\title{
Towards Two-Phase Scheduling of Real-time Applications in Distributed Systems
}

\author{
Mohammed I. Alghamdi ${ }^{\mathrm{a}}$, Xunfei Jiang ${ }^{\mathrm{b}, *}$, Ji Zhang $^{\mathrm{c}}$, Jifu Zhang ${ }^{\mathrm{d}}$, Minghua \\ Jiang $^{\mathrm{e}}$, Xiao Qin ${ }^{\mathrm{c}}$ \\ ${ }^{a}$ Department of Computer Science, Al-Baha University, Al-Baha City, Kingdom of Saudi \\ Arabia \\ ${ }^{b}$ Department of Computer Science, Earlham College, Indiana, USA 47374-4095 \\ ${ }^{c}$ Department of Computer Science and Software Engineering, Auburn University, \\ Alabama, USA 36849-5347 \\ ${ }^{d}$ School of Computer Science and Technology, Taiyuan University of Science and \\ Technology, Taiyuan 030024, China \\ ${ }^{e}$ College of Mathematics and Computer Science, Wuhan Textile University, Wuhan \\ 430073, China
}

\begin{abstract}
In this work we propose a two-phase scheduling technique (TOPS) for distributed real-time systems. Our TOPS scheduling approach has two distinct phases. The first phase is in charge of producing a scheduling sequence, whereas the second phase aims to dispatch tasks to computing nodes of a distributed system. The second phase also judiciously determines the starting time of each task. One salient feature of our approach lies in high flexibility, which allows system developers to apply multiple policies in each phase. The two phases are independent of one another; therefore, one can change a policy in one phase without configuring another phase. With TOPS in place, we are able to observe the impacts of sorting policies on the performance of scheduling policies. We implement a prototype of TOPS, where the first phase is comprised of three sorting policies, and the second phase consists of two scheduling policies. TOPS enables us to discover that combining the
\end{abstract}

\footnotetext{
${ }^{*}$ Corresponding author

Email addresses: mialmushilah@bu.edu.sa (Mohammed I. Alghamdi), jiangxu@earlham.edu (Xunfei Jiang), jizhang@auburn.edu (Ji Zhang), jifuzh@sina.com (Jifu Zhang), jmh@wtu.edu.cn (Minghua Jiang), xqin@auburn.edu (Xiao Qin)
}

Preprint submitted to Journal of Networking and Computer Application October 21, 2016

(C) 2017. This manuscript version is made available under the Elsevier user license http://www.elsevier.com/open-access/userlicense/1.0/ 
EDF (earliest deadline first) and AEAP (as early as possible) policies leads to an optimized performance among all six candidate algorithms.

Keywords: Run-time Systems, Task scheduling, Distributed Systems

\section{Introduction}

Cloud computing and service-oriented architectures are driving a shift toward distributed real-time systems, where the success of applications largely depends on quality-of-service (QoS) performance (i.e., deadlines and availability) [3]. A service level agreement (SLA) is applied as a contract between customers and service providers. In this study, we focus on scheduling techniques that allocate computing resources to tasks in a way to satisfy deadline requirements to fulfill a specified SLA in distributed systems (e.g., Hadoop computing environments [33]).

A distributed system consists of loosely coupled computing nodes connected through Ethernet or other networks. Scheduling schemes play an important role in achieving high performance of distributed systems [30]. Scheduling algorithms are divided into two categories, namely, dynamic scheduling [26][7] and static scheduling [8][9]. To satisfy a given SLA of real-time applications in distributed systems, scheduling mechanisms are responsible for ensuring that all tasks are complete before their deadline [34]. Existing real-time scheduling algorithms found in the literature include both preemptive [4] and non-preemptive [31]. A handful of real-time applications running in the service-oriented architecture paradigm are comprised of independent tasks (see, for example, [22]), where there is no intercommunication among real-time tasks.

Moreover, modern real-time tasks are no longer independent of another. Rather, a group of real-time tasks are collaborating to accomplish a common goal. A few real-time scheduling algorithms have been proposed to address the real-time scheduling issue in distributed systems [6]; unfortunately, these algorithms are inadequate for real-time tasks having precedence constraints. A growing number of real-time applications can be reasonably modelled in the form of a directed acyclic graph (DAG) [30][24], in which nodes and edges represent tasks and communication, respectively.

Third, breaking a scheduling mechanism into two distinct phases is appealing, because this approach allows us to investigate impacts of sorting policies on scheduling policies and vice versa. 
Therefore, we address the aforementioned three issues by developing a two-phase scheduling technique, or TOPS in short, for distributed real-time systems in this paper. Unlike traditional scheduling algorithms, TOPS seamlessly integrates two distinct phases [1]. The first phase is in charge of producing a scheduling sequence, whereas the second phase aims to dispatch the tasks to computing nodes of a distributed system. The second phase also judiciously determines the starting time of each task.

TOPS enables system developers to concurrently apply multiple policies in two phases. In other words, a system administrator is allowed to change a policy in one phase without configuring another phase. Given a distributed system where there are $M$ policies in phase one and $N$ policies in phase two, the total number of scheduling algorithms offered by the two-phase mechanism is $M N$.

A second benefit of this approach is making it possible to independently and concurrently investigate sorting policies in phase one and scheduling policies in phase two. With TOPS in place, we are able to observe the impacts of sorting policies on the performance of scheduling policies. We implement a prototype of TOPS, where the first phase is comprised of three sorting policies and the second phase consists of two scheduling policies. TOPS enables us to discover that combining the EDF and AEAP policies leads to an optimized performance among all the six candidate algorithms.

The main contributions of this work include:

1. an advanced two-phase scheduling framework (see Section 2).

2. the design and implementation of three sorting policies and two scheduling policies in TOPS (see Sections 4 and 5).

3. the performance evaluation of individual algorithms that integrate sorting and scheduling policies through extensive experiments (see Section 6).

The paper is organized as follows. In the next Section, we present a scheduling model for real-time tasks in addition to a novel scheduling mechanism. Section 3 describes the architecture and mechanism of the two-phase scheduling approach. Section 4 presents three sorting policies and Section 5 elaborates two scheduling policies implemented in TOPS. Testing methodology and performance analysis are presented in Section 6. Section 7 summarizes the related work of this study. Finally, conclusions are discussed in Section 8. 


\section{System Models and Problem Formulation}

In this section, we describe the task model as well as the system model for distributed real-time systems.

\subsection{Models}

A real-time application submitted to a distributed system is composed of inter-communicating tasks. We model each application in form of a real-time acyclic directed graph, where each real-time task is a fundamental component that is not divisible. Similar task models are widely employed in real-time systems [24][25][30].

A parallel and distributed real-time application is represented as a directed acyclic graph defined as $R G=\{T, E\}$, where task set $T$ contains a list of collaborative tasks (i.e., $T=\left\{t_{1}, t_{2}, \ldots, t_{n}\right\}$, and message set $E$ represents messages. Note that set $E$ also resembles precedence constraints among real-time tasks in $T$.

Task $t$ is modeled as a tuple, $t=(e, d, s, f, g, \rho)$, where $e, d, s$, and $f$ represent execution time, deadline, start time, and finish time of task $t . g$ is $t$ 's in-degree indicating the number of the task's parent tasks in task graph $R G$, and $\rho$ denotes a computing node to which $t$ is assigned.

Each message $m$ in $E$ is delivered from one task to another. We define message $m$ as a tuple, $m=(t s, t r, s, f, c)$, where $t s$ and $t r$ are tasks sending and receiving the message; $s$ and $f$ are the message's start time and finish time; and $c$ is message size determining the communication cost of the message.

In this study, we focus on parallel and distributed systems that have no global shared memory, meaning that each computing node independently manages its local main memory. A parallel and distributed system is formally expressed as a set $\Omega=\left\{P_{1}, P_{2}, \ldots, P_{m}\right\}$ of computing nodes, which form a fully interconnected computing environment. Each node in the system is modeled as $P_{i}=\left(\Delta_{i}, M_{i}\right)$, where tasks in list $\Delta_{i}$ are assigned to $P_{i}$ and tasks in $\Delta_{i}$ are sorted in a non-decreasing order of their start times; message set $M_{i}$ contains all messages delivered from node $P_{i}$ to the other nodes in the system. To facilitate the presentation, we model message set $M_{i}$ as a union of $m$ disjoint sets. Thus, we have $M i=M_{i 1} \cup \ldots \cup M_{i j} \cup \ldots \cup M_{i m}$, where $j \neq i$; all messages in set $M_{i j}$ are transmitted from node $P_{i}$ to $P_{j}$. The messages stored in $M_{i j}$ are sorted in non-decreasing order of message start times. It is worth noting 
that a vast majority of parallel and distributed systems (e.g., [19]) can be naturally represented by this system model.

\subsection{Scheduling Objectives}

A primary objective of scheduling tasks in a distributed real-time system is to assign each task to the most appropriate computing node and to decide the task's start time.

The scheduling objective for real-time applications running on distributed systems is two fold. First, a scheduler has to ensure that each task's real-time requirement is satisfied. The first objective can be formally expressed as

$$
\forall t_{i} \in \Delta_{j}, 1 \leq i \leq n, 1 \leq j \leq m: f_{i} \leq d_{i}
$$

Second, the system should minimize the number of active computing nodes to meet the deadlines of tasks. Before modelling this objective in a formal way, we denote a distributed system $\Omega$ as a union of two disjoint node sets $\Omega_{\text {active }}$ and $\Omega_{\text {idle }}$. Thus, we have $\Omega=\Omega_{\text {active }} \cup \Omega_{\text {idle }}$. $\Omega_{\text {active }}$ is a set of nodes to which there is at least one assigned task; $\Omega_{\text {idle }}$ contains nodes where there are no assigned tasks. Let $\left|\Omega_{\text {active }}\right|$ represent the number of computing nodes. Then, the second objective can be written as

$$
M P(R G, \Omega)=\min \left\{\left|\Omega_{\text {active }}\right|\right\}
$$

The benefits of the second objective is to improve system utilization while making large-scale distributed systems energy efficient. High energy efficiency can be straightforwardly achieved by placing idle nodes in set $\Omega_{\text {idle }}$ into the low-power mode.

\section{Two-Phase Scheduling}

In our work, we decompose an entire scheduling mechanism into two distinct phases. The first phase determines the most appropriate scheduling order by placing tasks into an ordered list; the second phase schedules tasks to the computing nodes of a distributed real-time system.

Fig. 1 depicts the framework of TOPS, where phase one manages a set $S O$ of $K$ sorting policies, $S O=O_{1}, O_{2}, \ldots, O_{K}$, and phase two handles a set $S S$ of $L$ scheduling policies, $S S=S_{1}, S_{2}, \ldots, S_{n}$.

The input parameter of an ordering policy $O_{i}$ is real-time task graph $R G$; the output of policy $O_{i}$ is an ordered list of tasks $O L_{i}=<t_{i 1}, t_{i 2},, t_{i n}>$. The 
input of the second phase is list $O L_{i}$ and the number of available computing nodes $m$. The job of the scheduling policy $S_{j}$ in the second phase is to judiciously allocate each task in $O L_{i}$ to a proper node, followed by determining the start times for tasks in $O L_{i}$. If the scheduling policy $S_{j}$ guarantees to complete all the real-time tasks before their specified deadlines, the scheduling result will be produced by phase two; otherwise, a warning message will be given.

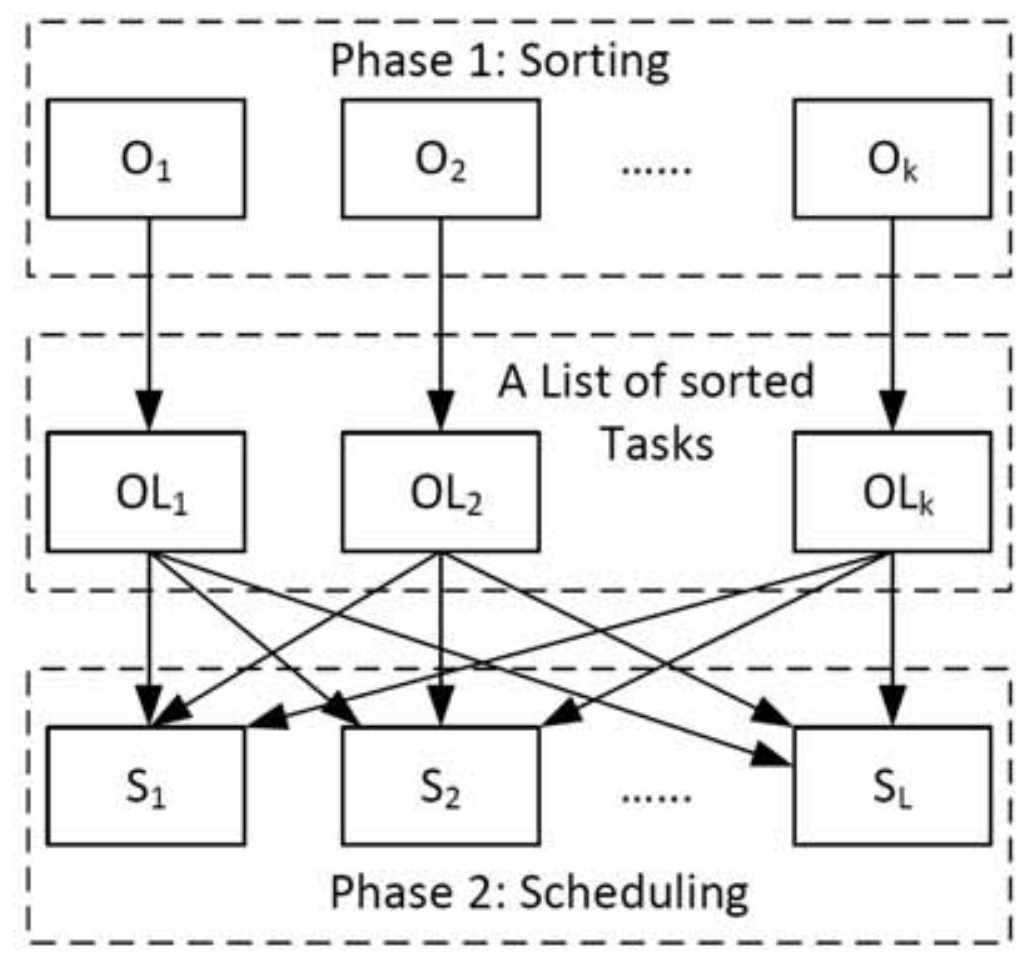

Figure 1: Decomposing an entire scheduling mechanism into two distinct phases. Phase 1 carries out a sorting policy; phase 2 performs a scheduling policy. Phase 1 manages a set $S O$ of $K$ sorting policies (i.e., $S O=O_{1}, O_{2}, \ldots, O_{K}$ ), whereas phase 2 handles a set $S S$ of $L$ scheduling policies (i.e., $S S=S_{1}, S_{2}, \ldots, S_{L}$ ). Each sorting policy (e.g., $O_{i}$ ) yields a distinct sorted list of tasks (e.g., $O L_{i}$ ). Each ordered list (e.g., $O L_{i}$ can be applied as an input to all the available scheduling policies (i.e., from $S_{1}$ to $S_{L}$ ).

It is worth mentioning that given a real-time task graph, each sorting policy (e.g., $O_{i}$ ) yields a distinct sorted list of tasks (e.g., $O L_{i}$ ). A ordered list such as $O L_{i}$ can be configured as an input to any chosen scheduling policy from all the candidate policies (i.e., from policy $S_{1}$ to policy $S_{L}$ ).

A scheduling policy is unable to meet real-time requirements of appli- 
cations due to two potential reasons. First, the limited number of available computing nodes may result in the failure of scheduling. To solve the scheduling problem induced by the lack of computing resources, one can simply extend the distributed system by adding more nodes. Second, increasing the number of nodes might not be enough to guarantee the deadlines of real-time tasks in $R G$. In this case, it is the responsibility of application developers to redesign their applications, focusing on task collaborations as well as the corresponding real-time constraints. Note that the topic of how to design a schedulable real-time application (i.e., $R G$ ) is out the scope of our study. In this paper, we focus on real-time applications that have a schedulable task graph $R G$. In other words, the applications considered in this research can have their deadline requirements met if a sufficient number of computing resources are offered.

The goal of TOPS is to make it feasible to independently and concurrently investigate sorting policies and scheduling policies in a scheduling mechanism. In light of TOPS, one is capable of observing the impacts of sorting policies on the performance of scheduling policies and vice versa.

We implement a prototype of TOPS, where system administrators can install and disable sorting and scheduling policies. TOPS is formally modeled as $T O P S=S O, O L, S S$, where $S O$ is a set of sorting policies, $S S$ is a set of scheduling policies, and $O L$ is ordered list of tasks. Given a distributed system where there are $K$ policies in phase one and $L$ policies in phase two, the total number of scheduling algorithms offered by the two-phase mechanism TOPS is $K L$.

\section{Phase One: Sorting Policies}

Three candidate sorting policies were considered in the first phase of TOPS. Property 1 was used to validate that a scheduling sequence yielded by a sorting policy is accurate.

$$
\begin{aligned}
\text { Property } 1: \forall t_{j}, t_{k} \in T & {\left[\left(t_{j}, t_{k}\right) \in E \rightarrow\right.} \\
& \left.\left(\exists t_{i a}, t_{i b} \in O L_{i}\right) \wedge\left(t_{i a}=t_{j}, t_{i b}=t_{k}\right) \wedge(a<b)\right] .
\end{aligned}
$$

The above property states that if task $t_{j}$ transmits data to task $t_{k}$, then task $t_{j}$ must be scheduled prior to the scheduling of task $t_{k}$. The property indicates that precedence constraints in an application $R G$ has to be enforced by a sorting policy in TOPS. 
We present three sorting policies, each of which produces an ordered list $O L$ of tasks; each list $O L$ satisfies property 1. These three policies sort tasks in set $T$ according to their in-degrees; the in-degree value of each task $t$ relies on the number of $t$ 's unsorted parent tasks. For example, if one parent task of $t$ has been placed into list $O L$, then the $g$ value of $t$ will be decreased by one. Only when $t$ 's $g$ value drops down to zero, can the sorting policies insert task $t$ into $O L$. In doing so, it is guaranteed that before $t$ is placed into list $O L$, for all $t$ 's parent tasks have already been sorted and stored into $O L$. In other words, list $O L$ created by any sorting policy can ensure that task $t$ is scheduled after all its parent tasks have been scheduled.

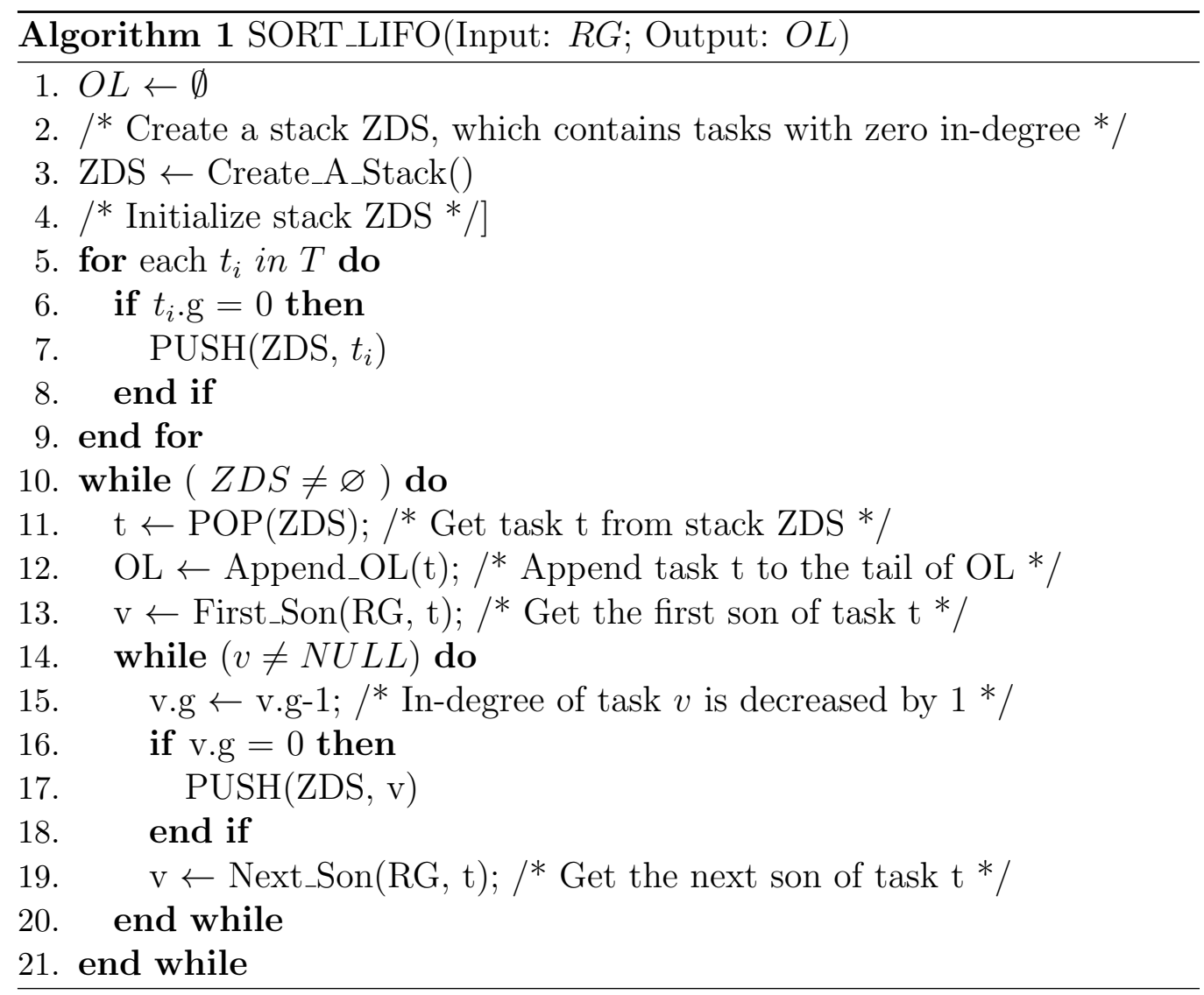

Among the three policies, the SORT_LIFO and SORT_FIFO policies are quite similar. SORT_LIFO sorting policy (as shown in Algorithm 1) leverages a stack to store all tasks whose in-degree values are zero. SORT_FIFO (as shown in Algorithm 2) stores tasks with zero in-degree values into a queue 
rather than a stack.

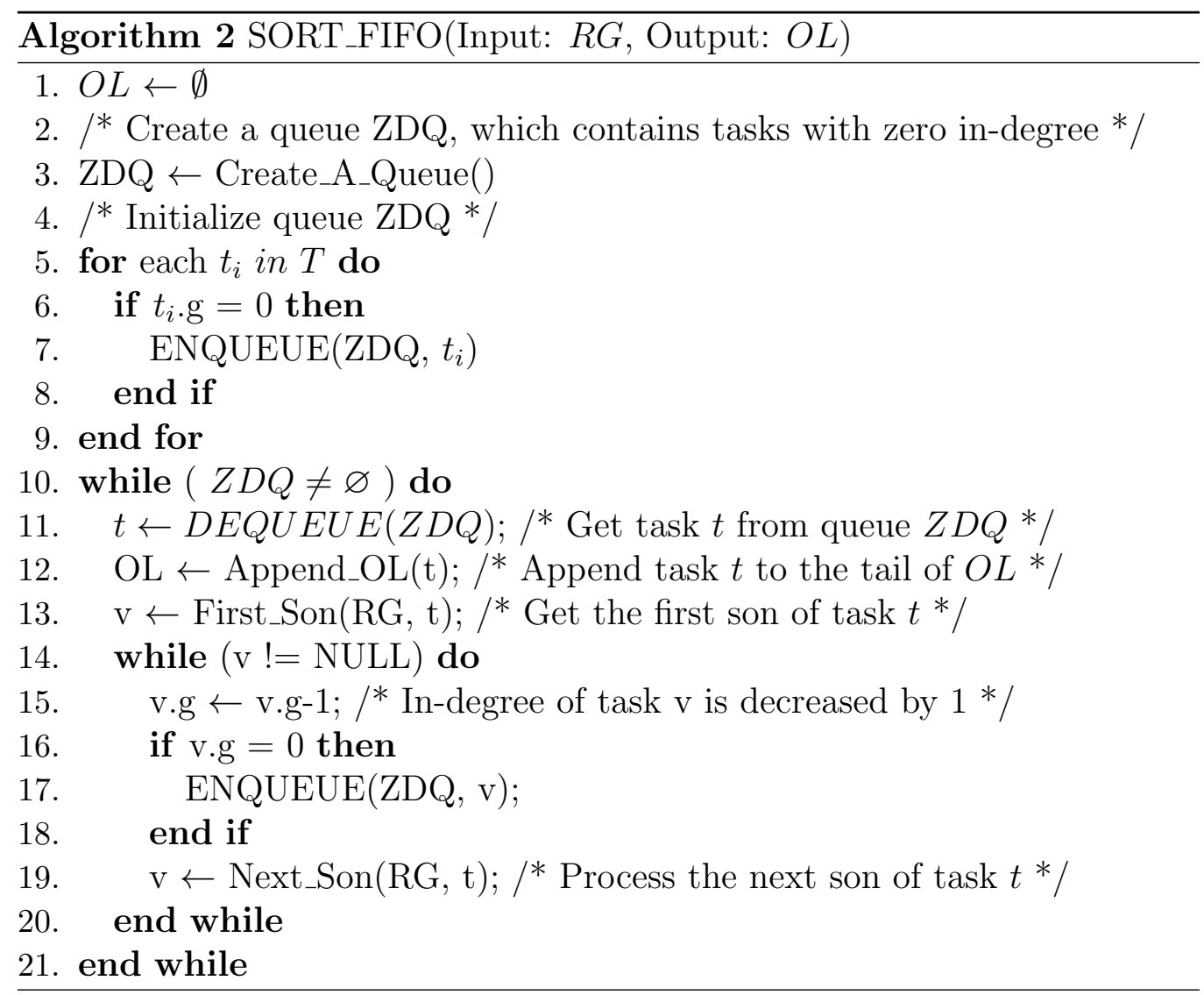

Algorithm 3 shows the pseudo-code of SORT_EDF, which is the third sorting policy implemented in TOPS. The SORT_EDF temporarily stores tasks with a zero in-degree value into a list (i.e., $Z D L)$. SORT_EDF sequentially removes one task at a time from list $Z D L$ and appends the task to the end of list $O L$. When SORT_EDF chooses a candidate task to move from list $Z D L$ into $O L$, a task with the earliest deadline is given the highest priority. Such a strategy of selecting a candidate task is formally expressed by Property 2.

Property 2: When a task $\mathrm{t}$ is moved from list ZDL to list OL, the following statement holds: $\forall t_{i} \in Z D L: t . d \leq t_{i} . d$ 


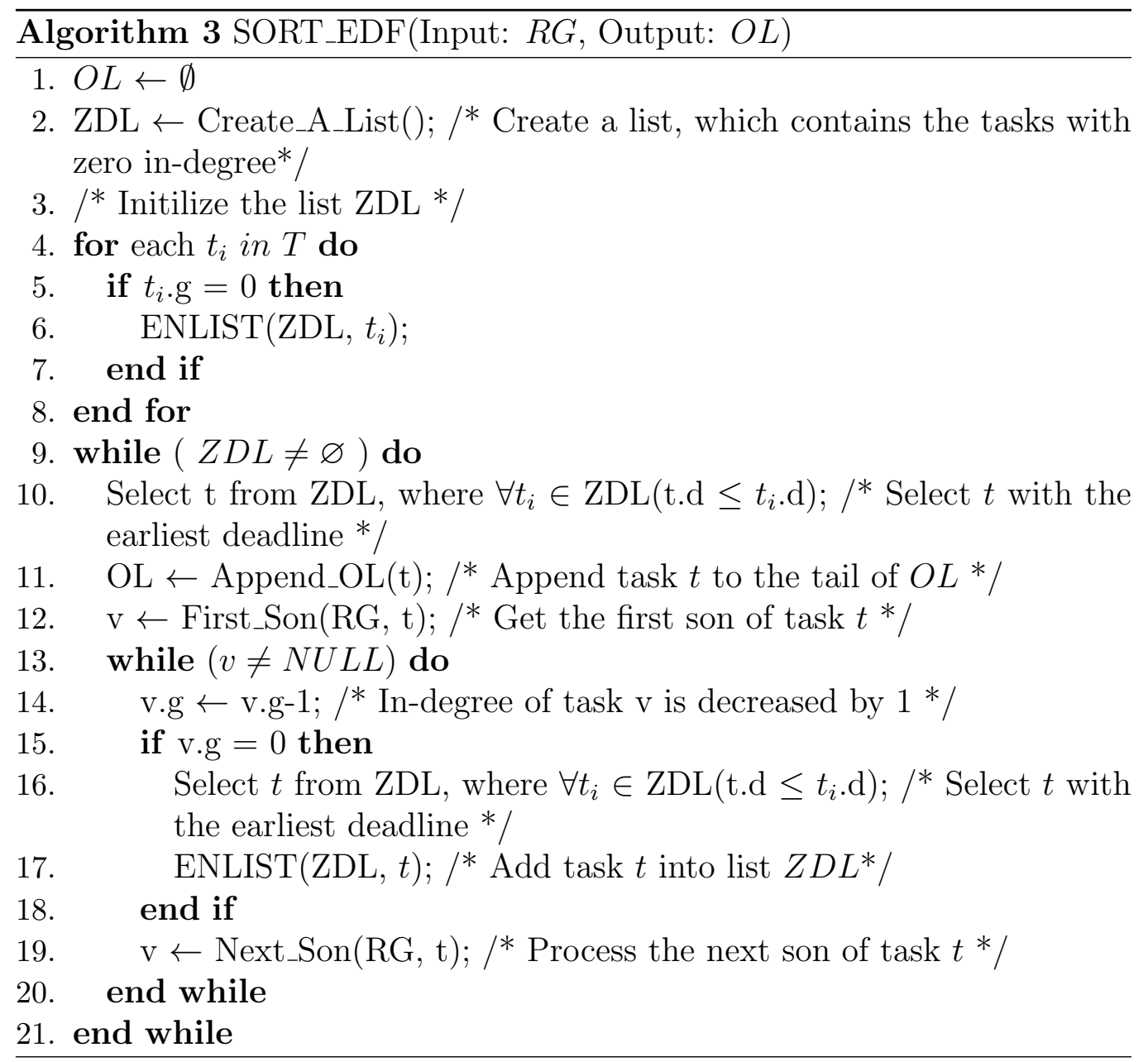

\section{Phase Two: Scheduling Policies}

After the sorting phase is accomplished in TOPS, a task graph $R G$ is successfully transformed into a sequence list, where tasks are waiting to be dispatched by a scheduling policy in the second phase of TOPS. In this Section, we describe two scheduling policies incorporated in phase two of TOPS.

A scheduling policy in this phase is used to allocate real-time tasks to processors in the system and to decide the start time of each task. In order to compute start times, we have to calculate the earliest start time or EST of a task on each computing node. EST can be derived from the earliest available 
time EAT, which is in turn obtained from the finish time of the task's parent tasks and its message arrival times. In this study, if task $t$ and one of its parent tasks $t_{p}$ are allocated to the same node, then the communication time of transmitting a message from $t$ to $t_{p}$ becomes negligible. If two tasks $t$ and $t_{p}$ are assigned to two different nodes, their message arrival time will be determined by the finish time of parent task $t_{p}$, message size, and available network bandwidth. Algorithm 4 is applied to obtain the start time of a message $m$ being sent from node $P_{i}$ to node $P_{j}$.

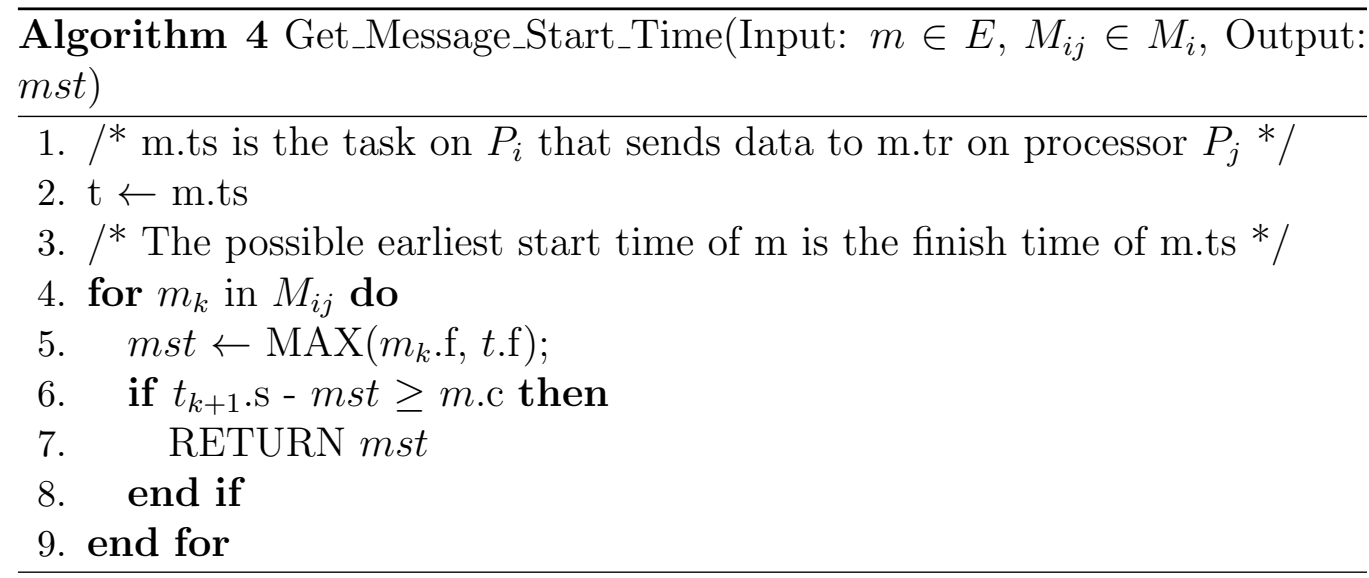

Algorithm 4 computes the start time for a message $m$ delivered from $P_{i}$ to $P_{j}$. EAT of a task $t_{i}$ on node $P_{j}$ can be generated by Algorithm 4. Let $M S\left(t_{i}\right)$ be a set of messages being transmitted to task $t_{i}$; we formally express $M S\left(t_{i}\right)$ as

$$
M S\left(t_{i}\right)=\left\{m \in E \mid m . t r=t_{i}\right\}
$$

Algorithm 5 aims to compute the earliest available time of task $t_{i}$ on node $P_{j}$. This algorithm first determines the start time of each message in set $M S\left(t_{i}\right)$ (see also (5)), then calculates the finish time of each message. EAT of task $t_{i}$ is equal to the latest finish time in $M S\left(t_{i}\right)$.

Now let us consider task $t$ and its candidate node $p$. If node $p$ has the earliest idle time slot starting later than $t$ s EAT and also large enough to execute task $t$, then task $t$ can be allocated to node $p$, on which $t$ starts in an idle time slot. Algorithm 6 is designed to facilitate the computation of the earliest start time for task $t_{i}$ on node $P_{j}$. Since all tasks in set $\Delta$ are sorted in a non-decreasing order of start time, Algorithm 6 checks all the idle time slots on node $P_{j}$, starting from the earliest idle slot to the latest one. The 

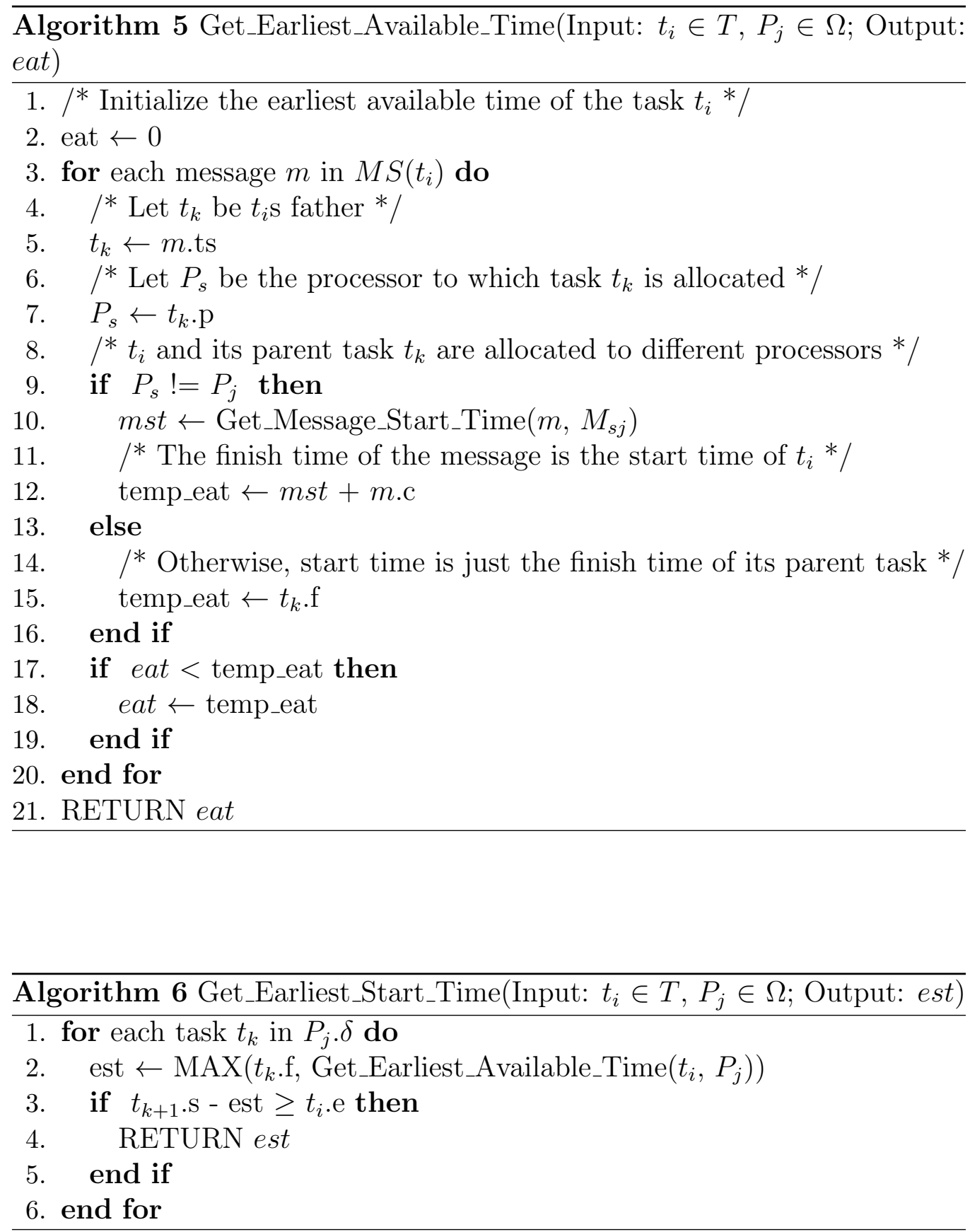
Get_Earliest_Start_Time algorithm terminates the procedure of idle-time-slot checking and returns the earliest start time after an appropriate idle time slot.

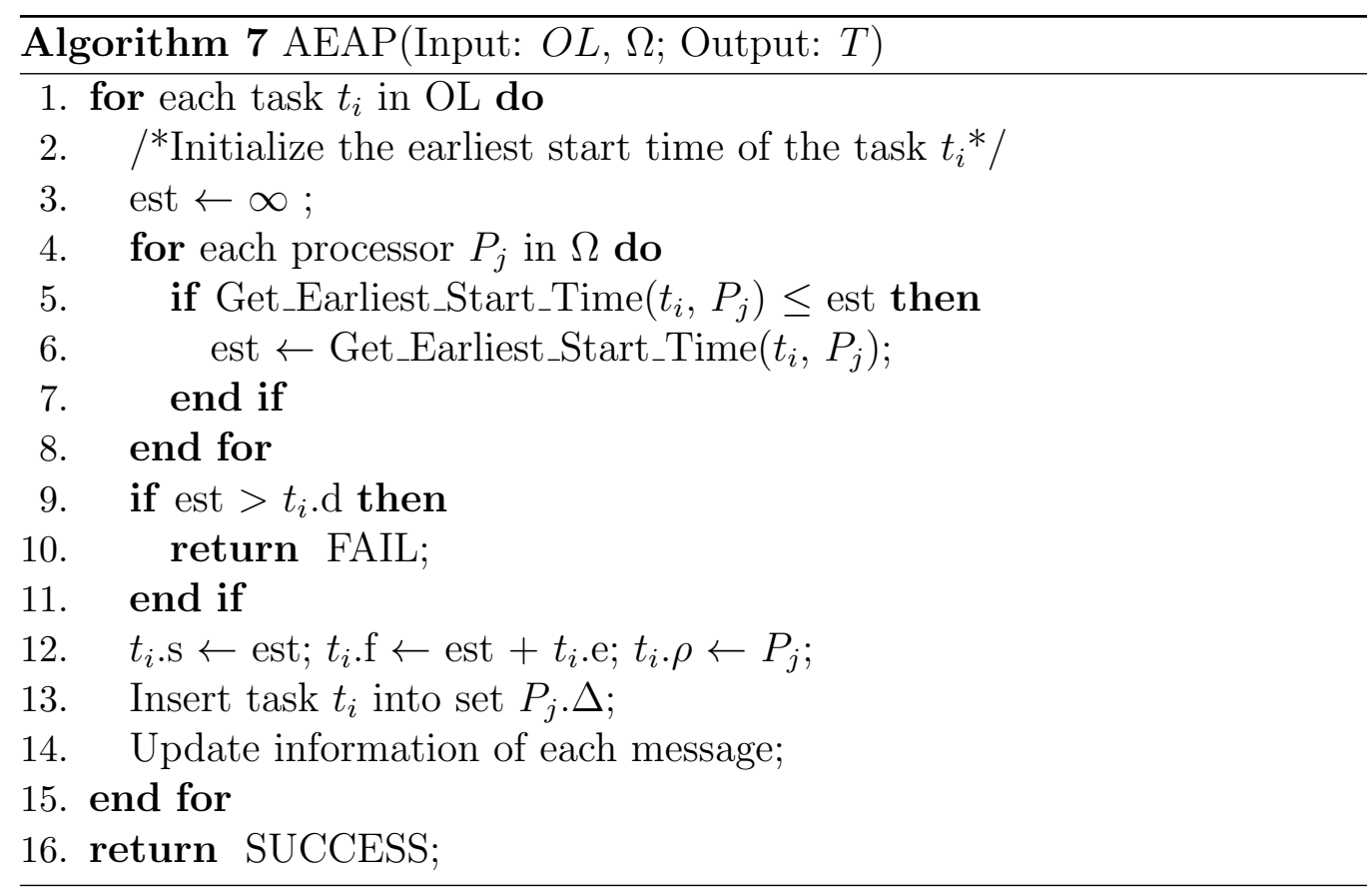

Now we are positioned to seamlessly integrate the aforementioned three algorithms (i.e, Algorithms 4, 5, and 6) to implement the first scheduling policy in the second phase of TOPS. Algorithm 7 (i.e., AEAP - schedule as early as possible) carries out the following step to make scheduling decisions for real-time tasks. Given real-time task $t_{i}$ and a distributed system, AEAP computes the EST value on each candidate node in the distributed system; chooses a candidate node that offers task $t_{i}$ the earliest EST value; determines whether task $t_{i}$ 's start time is earlier than its specified deadline. A warning message is returned if $t_{i}$ 's deadline is violated; such a violation terminates the scheduling procedure.

The second scheduling policy called ALAP (i.e., schedule as late as possible). Under a condition that the deadlines of all real-time tasks can be met, ALAP starts the tasks as late as possible. Before presenting ALAP, we describe a procedure called Get_Latest_Start_time to calculate the latest start time or LST of task $t_{i}$ on node $P_{j}$. This procedure examines all idle time slots on node $P_{j}$ by computing the earliest start time and the latest possible finish time or LPFT. An idle time slot is considered as a candidate idle time slot 
if time between EST and LPFT is sufficiently large to accommodate task $t_{i}$. Among all the candidate time slots, the procedure chooses the latest one and decides the latest start time. Algorithm 8 outlines the Get_Latest_Start_time procedure.

Algorithm 8 Get_Latest_Start_Time(Input: $t_{i} \in \mathrm{T}, P_{j} \in \Omega$; Output: lst, find)

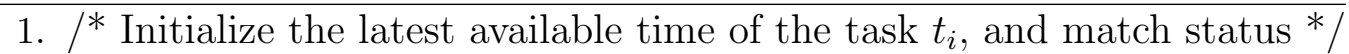

2. lst $\leftarrow 0$; find $\leftarrow \mathrm{NO}$;

3. eat $\leftarrow$ Get_Earliest_Available_Time $\left(t_{i}, P_{j}\right)$;

4. for each task $t_{k}$ in $P_{j} . \Delta$ do

5. $\quad / *$ Compute the earliest start time in the idle time slot */

6. $\quad$ est $\leftarrow \operatorname{MAX}\left(t_{k}\right.$.f, eat $)$;

7. $\quad / *$ Compute the latest possible finish time in the idle time slot */

8. $\quad \operatorname{lpft} \leftarrow \operatorname{MIN}\left(t_{k+1} \cdot \mathrm{s}, t_{i} \cdot \mathrm{d}\right)$;

9. $\quad / *$ If idle time is large enough to accommodate task $t_{i} * /$

10. if lpft - est $\geq t_{i}$. e then

11. $/ *$ Compute the start time in this idle time slot*/

12. $\quad$ find $\leftarrow$ YES; st $\leftarrow$ lpft $-t_{i}$.e;

13. if $\mathrm{st}>$ lst then

14. $\quad$ lst $\leftarrow$ st

15. end if

16. end if

17. end for

18. return (lst, find);

Algorithm 9 shows the pseudo-code of the ALAP scheduling policy, which takes the following steps to generate scheduling decisions. ALAP first decides the LST of each task (e.g., $t_{i}$ ) on each node (e.g., $p_{j}$ ) in the distributed system. Then, ALAP selects a node on which $t_{i}$ exhibits the latest LST value. If such a candidate node can not be found, ALAP will inform users that the given task set $R G$ is unschedulable.

\section{Experimental Results}

In this section, we demonstrate that TOPS enables system administrators to investigate performance impact of a sorting policy and a scheduling policy to quickly identify the best combination of a policy in phase one and 


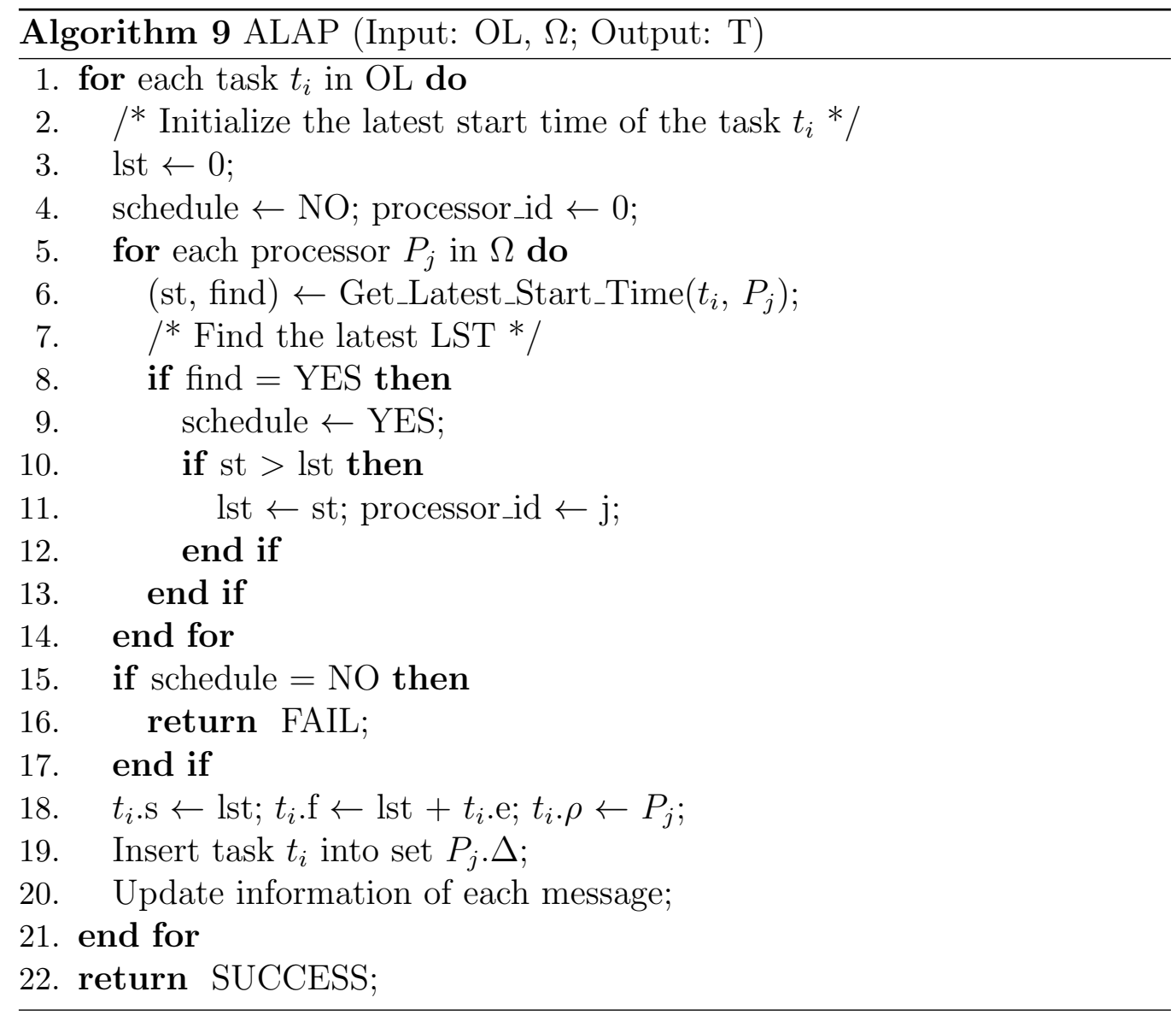

a second policy in phase two. We implement six algorithms by integrating the three policies in phase one with the two policies in phase two. This section presents the performance results of schedules generated by the six algorithms in TOPS. We name the six algorithms as FIFO/AEAP, FIFO/ALAP, FILO/AEAP, FILO/ALAP, EDF/AEAP, and EDF/ALAP.

\subsection{Experimental Setup}

We implement the simulator on a server, which contains an Intel(R) E5800 @ 3.2GHz CPU, 4GB DDR3 memory, and Intel G41 Chipset. The operating system running in the server is Ubuntu 10.04 X86 64 (Kernel 2.6.32). We implement a task graph generator to create two types of real-time applications, which are commonly used to study a wide variety of schedulers developed for real-world distributed systems [11]. Fig. 2 shows the two task graphs, 
namely, binary trees and lattices.
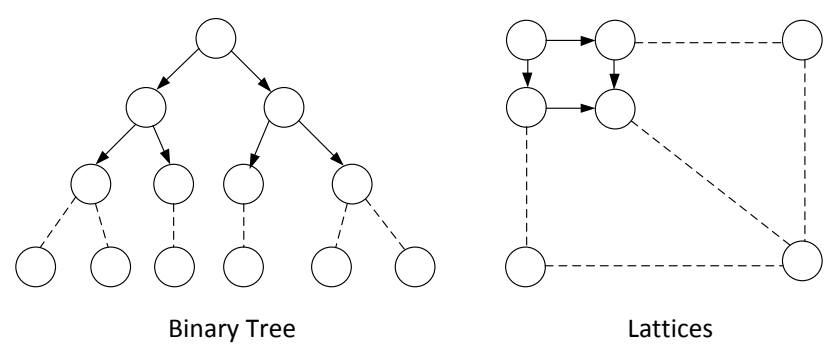

Figure 2: Two types of real-time applications.

We introduce two parameters - DER and CCR - to configure workload conditions. DER is a deadline-to-execution-time ratio, whereas CCR is a communication-computation ratio [16]. For each type of real-time application, the DER value is set to $1.6,2$, and 10 , respectively; CCR is set to be 0.1. A DER value of 1.6 represents an application with urgent deadlines; a DER value of 10 resembles an application with loose deadlines; and a DER value of 2 represents an application between the two extreme ends of the spectrum (i.e., deadlines are neither too urgent nor too loose). The value of CCR of 0.1 is a computation intensive graph.

Real-time applications to be scheduled by TOPS are generated by carrying out the following steps. First, the execution time of each task is randomly chosen according to a uniform distribution with a mean equal to 100 . The deadline of each task is derived as a product of its execution time and the DER value. Second, we randomly selected communication time for each message with a mean value of $100 * C C R$. We also increased the number of tasks in a range from 10 to 90 with an increment of 10.

\subsection{Minimum Number of Nodes}

After given a set of real-time tasks and the number of computing nodes $k$, the scheduler in TOPS is able to determine whether $k$ nodes can accomplish all the tasks before their deadlines. Algorithm 10, called Find_MNN or Find Minimum Number of Nodes, is conducive to optimizing the minimum number of nodes that are capable of completing all the tasks before their specified deadlines. Input parameter Sort_Alg indicates a sorting policy generating list $O L$ in the first phase of TOPS, and input parameter Sch_Alg specifies a scheduling policy managing to schedule the task set. 
In theory, the initial upper bound (i.e., $n$ in Algorithm Find_MNN) can be configured in two ways. First, the initial value of the upper bound is set to be the number of computing node in an existing system. Second, in case that a computing system does not exist to suggest the upper bound's initial value, the upper bound will be configured to a number that is sufficiently large to accommodate all tasks. For example, the upper bound equals to the number $n$ of tasks in task set $T$. In our experiments, we take the second approach, because we pay attention to minimize the number of nodes without targeting at a specific computing system. The pseudo-code of Find_MNN is illustrated in Algorithm 10.

Algorithm 10 Find_MNN: Find Minimum Number of Nodes(Input: $R G$, $\Omega$, Sort_Alg, Sch_Alg; Output: $\mathrm{k}, \Omega$ )

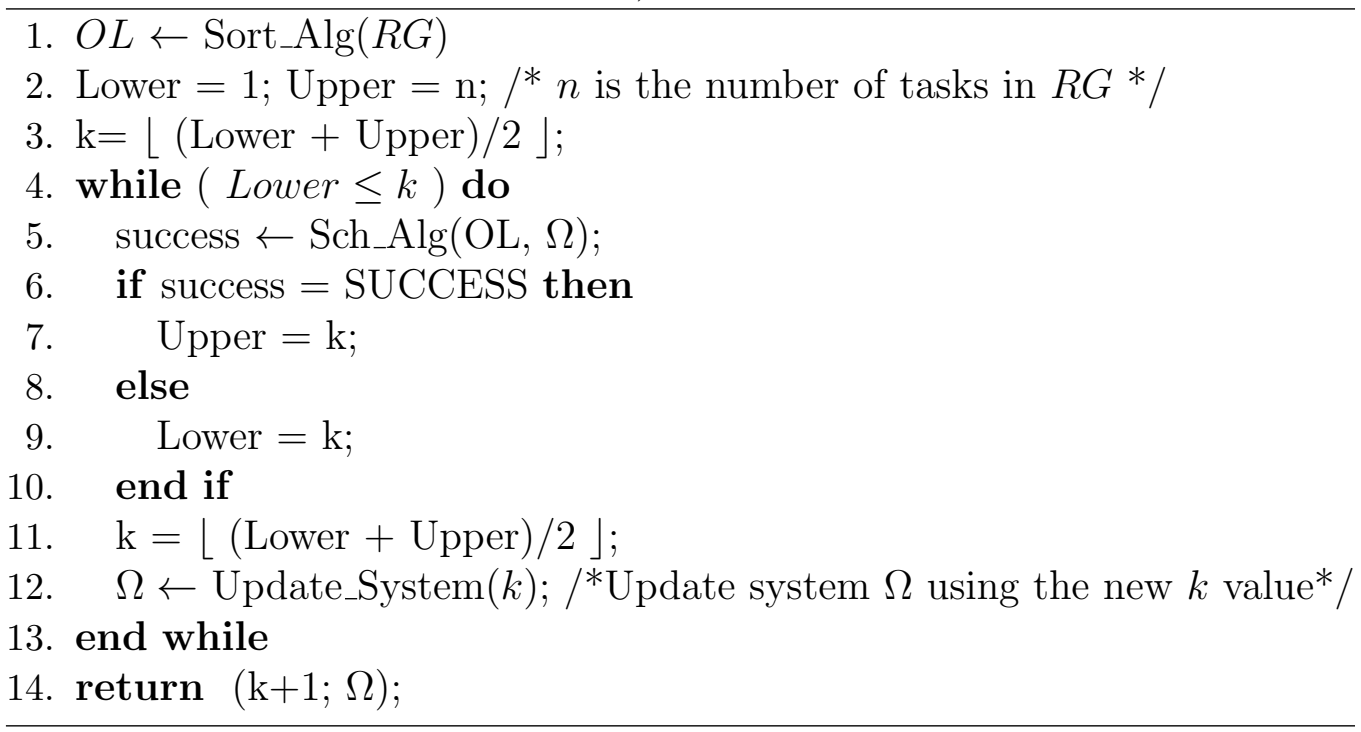

Algorithm 10 performs a binary search for the minimum $k$ value. More specifically, Algorithm 10 iteratively and progressively narrow the search range expressed by the two index limits (i.e., Lower and Upper), which are initialized to be 1 and $n$ (i.e., the number of tasks in task graph $R G$ ) in Step 2. During each iteration, a new search range is updated by determining a midpoint, which is calculated in Step 11 as $\lfloor($ Lower $+U p p e r) / 2\rfloor$. If Step 5 confirms that the previous $k$ value is sufficiently large to guarantee the deadlines of the tasks in $R G$, then the new search range is set from the previous Lower to the midpoint (i.e., $k$ ). Otherwise, the new range range is from the midpoint to the previous Upper. 


\subsection{Number of Tasks}

In the first group of experiments, we evaluate the impact of the number of tasks on the algorithms scheduling performance measured in terms of the minimum number of nodes. We quantitatively compare the performance of all the six schedulers developed using our TOPS framework. Fig. 3 shows the scheduling results for applications modeled as binary trees, in which the deadline-to-execution-time ratio (i.e., DER) and the communication-timeto-execution time ratio (i.e., CCR) are 2.1 and 0.1 , respectively. Fig. 3 reveals that for all the tested schedulers, the number of real-time tasks in an application make a significant impact on the minimum number of nodes. More specifically, the minimum number of nodes increases when the number of tasks goes up. Such a performance trend can be attributed to the fact that scaling up the application size forces computing resources to be expended to meet tasks' real-time constraints.

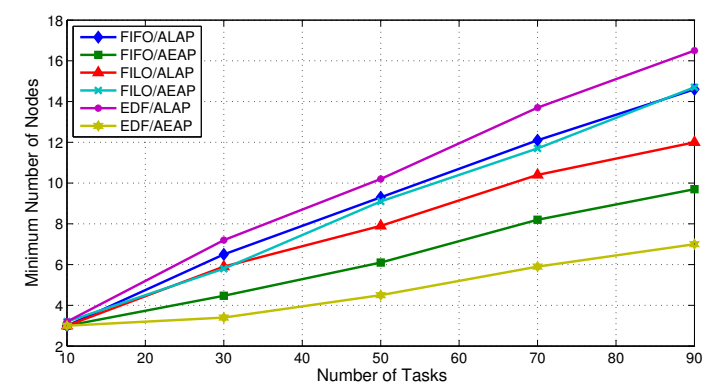

Figure 3: Average minimum number of nodes determined by the six algorithms. $\mathrm{CCR}=0.1$, $\mathrm{DER}=2.1$

We observe from Fig. 3 that among all the tested schedulers, EDF/AEAP is the best scheduling solution and EDF/ALAP is the worst one. The scheduling performance of FIFO/ALAP and FILO/AEAP are quite similar. ALAP and AEAP have their own merits under various workload conditions. If in the first phase SORT_FIFO or SORT_EDF is employed to produce list $O L$, then applying the AEAP policy in the second phase is an optimal choice. Thus, we draw two conclusions from this group of experiments: (1) FIFO/AEAP is superior to FIFO/ALAP, and (2) EDF/AEAP outperforms EDF/ALAP. Interestingly, when the SORT_FILO policy is invoked in the first phase of TOPS, then the evidence shows that ALAP is superior to AEAP in the second phase. 


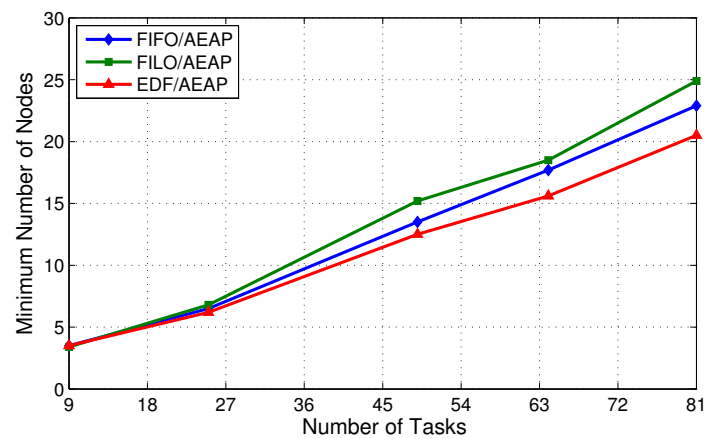

Figure 4: Average minimum number of nodes determined by the AEAP algorithms. $\mathrm{CCR}=0.1, \mathrm{DER}=1.6$

When we test applications that are represented as lattice graphs, we observe that the ALAP policy is unable to meet real-time requirements due to its poor scheduling performance. Our experimental results show that when it comes to the lattice case, the AEAP's performance is a whole lot better than that of ALAP. We omit the results of ALAP from Fig. 4 due to its poor performance; we only demonstrate the performance of the AEAP algorithms in Fig. 4. Fig. 4 confirms that among the tested schedulers, EDF/AEAP is the best algorithm and FILO/AEAP has the worst performance. These experimental results are consistent with those plotted in Fig. 3. We conclude that if the AEAP policy is adopted in the second phase of TOPS, then the SORT_EDF policy in the first phase tends to enhance the real-time performance of parallel and distributed systems. When it comes to largescale applications composed of an excessive number of real-time tasks, the performance improvement offered by SORT_EDF becomes more pronounced.

\subsection{Deadlines}

Now we study the impact of deadlines on the performance of TOPS. We configure the deadline parameter by setting the deadline-to-execution-time ratio or DER to 1.6, 2, and 10, respectively. We investigate how DER affects the minimum number of nodes generated by the tested schedulers. Figs. 5 and 6 plot the experimental results of real-time applications modeled in form of binary trees and lattices, respectively.

Fig. 5 omits the results of FILO/AEAP, because FIFO/ALAP and FILO/AEAP share similar scheduling performance. First, EDF/AEAP algorithm outperforms all the other tested algorithms. Second, the performance improvement 


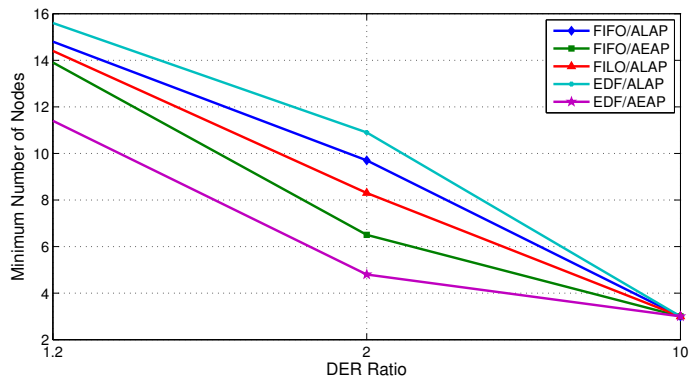

Figure 5: Relation between the DER ratio and the average minimum number of nodes for the five algorithms. Applications are modeled as binary trees, where CCR is 0.1 and the number of tasks is 50 .

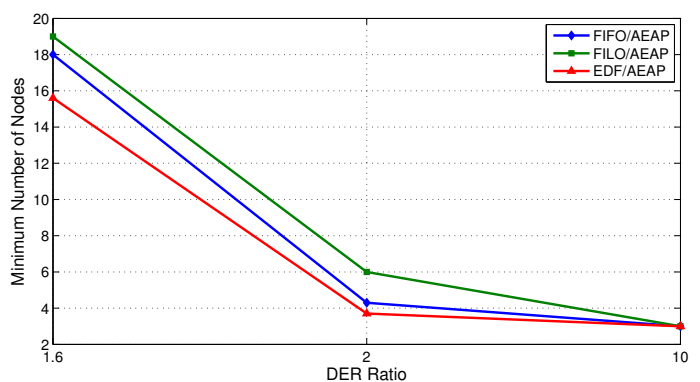

Figure 6: Relation between the DER ratio and the average minimum number of nodes for the three algorithms. Applications are modeled as lattices, where CCR is 0.1 and the number of tasks is 64 .

offered by EDF/AEAP is diminishing when the DER value increases; if DER is a large value (e.g., larger than ten), all the algorithms exhibit similar performance in terms of minimum number of nodes. This trend is reasonable, because a large DER value leads to loose deadlines, which in turn make scheduling length a non-critical issue. In such a case, even a limited number of computing nodes are capable of satisfying the real-time requirements of all tasks. In contrast, a small DER value implies tight deadlines of tasks, which rely on well-designed schedulers to shorten scheduling lengths on computing nodes. The SORT_EDF policy inherently gives the highest priority to tasks with the earliest deadline, thereby scheduling task with urgent deadlines earlier than other non-urgent tasks. Urgent tasks should be considered earlier, because scheduling tasks with tight deadlines later is very likely to cause the deadline to be missed. 


\subsection{Communication Computation Ratio (CCR)}

Now we conduct a group of experiments to study the impact of communicationcomputation ratio or $C C R$ on the performance of TOPS. Specifically, we investigate how CCR affects the minimum number of nodes generated by the tested schedulers. Again, we choose binary trees as a representative communication patterns in real-time applications. In the experiments, $C C R$ is set to $0.1,0.5,1,2,10$, respectively. Figs. 7 plots the results of real-time applications. The results when CCR is larger than 10 are omitted from the figure, because the task graphs are so communication-intensive that the minimum number of nodes are greater than 50 .

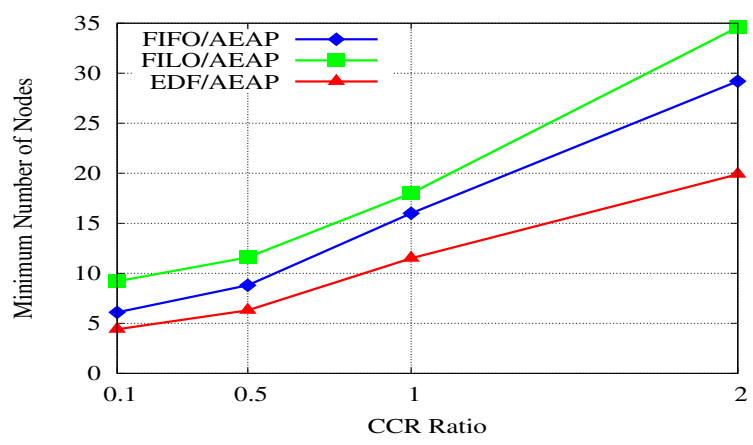

Figure 7: Impacts of the communication-computation ratio (CCR) on the average minimum number of nodes for the three algorithms. Applications are modeled as binary trees, where DER is 2.1 and the number of tasks is 50 .

As shown in Fig. 7, when the CCR ratio is increasing, the minimum number of nodes required to complete the tasks prior to their deadlines is also going up. A large CCR indicates high communication overhead, which in turn results in long execution times. When the execution times grow, the schedulers have to rely on an increased number of computing nodes to guarantee the deadlines. Comparing the scheduling results of the three schedulers, we observe that EDF/AEAP is the still superior to the other two competitors; again, FILO/AEAP delivers the worst performance. The experimental results plotted in Fig. 7 are consistent with those discussed in the previous subsections.

\section{Related Work}

Scheduling algorithms can be classified into two categories, namely, static and dynamic [14][35]. Static scheduling algorithms make scheduling deci- 
sions prior to the submission of tasks to a computing system [27]. Dynamic scheduling algorithms are applied to schedule tasks whose arrival times are not known a priori (see, for example, [12]). In this study, we focus on the development of TOPS, in which two independent phases are integrated to form a static real-time scheduler. It is worth mentioning that our TOPS framework can be extended to dynamically schedule real-time tasks. We will address the dynamic scheduling issue in one of our future studies.

Some existing scheduler employ preemptive scheduling policies. All the schedulers implemented using TOPS in this study are non-preemptive in nature; thus, an executing real-time task cannot be preempted by another task. Non-preemptive scheduling is efficient in the context of static schedulers, because non-preemption helps in reducing overhead caused by switching among tasks [20][21]. As such, the evaluated schedulers in this study take the nonpreemptive scheduling approach.

Venugopalan and Sinnen developed a mixed integer linear programming solution or MILP to address the scheduling problem in multiprocessor systems [32]. Lombardi et al. proposed an approach to tackle predictable and efficient nonpreemptive scheduling of multitask applications in the presence of duration uncertainty [18]. Their approach does not require probability distributions to be known in advance, and rely on simple information (bounds, average values). Cordasco et al. introduced a new quality metric, AREA, for schedules that execute computations having interdependent constituent chores (jobs, tasks, etc.) on modern computing platforms which exhibit dynamic heterogeneity [10]. On these platforms, availability and computing power of their constituent resources can change unexpectedly and dynamically. They measured the average number of chores that a schedule renders eligible for execution at each step of a computation. In a recent study, $\mathrm{Li}$ et al. investigated stochastic scheduling attributes and methods to deal with various random variables in scheduling stochastic tasks [17]. They designed a stochastic dynamic level scheduling algorithm to efficiently schedule precedence constrained stochastic tasks. Palmieri et al. proposed a novel uncoordinated fully distributed scheduling system for federated cloud organizations [23]. Their solution is based on independent, competing, and self-interested job/task execution agents, driven by optimum social welfare criteria. The aforementioned scheduling strategies are able to achieve high performance for non-real-time applications. In contrast, the goal of TOPS is to develop and analyze schedulers for real-time applications. The TOPS schedulers guarantee to complete real-time tasks within their deadlines. 
Beaumont et al. addressed the issue of assigning a set of clients with demands to a set of servers with degree and capacities constraints [2]. They designed an allocation scheme to maximizing the overall system throughput in such a way that the number of clients assigned to a server is smaller than the server's degree and their overall demand is smaller than the server's capacity. Leal et al. proposed a decentralized model in the context of scheduling independent tasks in Federated Grids [15]. They also developed a set of meta-schedulers, each of which implements a mapping strategy to shorten makespan and optimize resource performance. The main axis of these two studies is that they solve the scheduling problem of independent tasks; this problem has several natural applications in the context of virtual machines allocation. Unlike the prior work, the algorithms built from our TOPS have an inherent capability of scheduling real-time tasks with precedence contraints.

Jansen and Zhang developed an improved approximation algorithm for scheduling malleable tasks under general precedence constraints, with a ratio at most 4.73 makespan (maximum completion time) [13]. Socci et al. studied multiprocessor scheduling problem of a finite set of precedence-related mixed criticality jobs. They observed that the mixed-criticality increases the complication of real time scheduling, since some subsystems are safetycritical (e.g., car braking system); while others are not (e.g., car digital radio). With the assumption, a novel method was proposed to improve the schedulability for mixed-criticality setting by modifying fixed-priority assignments for jobs [28]. Chen et al. developed a heterogeneous scheduling framework for dynamic workloads with real-time and precedence constraints [5]. In their design, a general purpose processor core and a synergistic processor core are dedicated to separate schedulers with different scheduling policies. Stavrinides and Karatza proposed a novel scheduling solution that combines imprecise computations and bin packing strategies to improve performance of real-time heterogeneous clusters [29]. Their scheme achieves good scheduling performance by exploiting schedule holes while making tradeoffs between precision and timeliness. A distinct difference between our TOPS and the aforementioned approaches is that these methods are focused on real-time scheduling for heterogeneous computing environments, whereas TOPS aims at a flexible way of adjust a sorting policy in the first phase without configuring a scheduling policy in the second phase. 


\section{Conclusion}

In this paper, we proposed TOPS - a two-phase scheduling approach for parallel and distributed real-time systems. TOPS decomposes a scheduler into two main phases. The first phase is a sorting policy, whereas the second phase is a scheduling policy. With TOPS in place, one is allowed to study the impact of a sorting policy on a scheduling policy and vice versa. TOPS makes it possible to flexibly create a wide range of schedulers by seamlessly integrating a sorting policy in phase one with a scheduling policy in phase two. To demonstrate the strength of TOPS, we designed three sorting policies in the first phase and two scheduling policies in the second phase. We quantitatively evaluate the performance of the six schedulers, which incorporate the sorting policies and scheduling policies in the TOPS framework. Our experimental results show that among the tested algorithms, EDF/AEAP exhibits the best scheduling performance.

Further research is needed to extend TOPS to address the scheduling issues in heterogeneous systems, where there is more than one type of computing node. We plan to investigate the applicability of TOPS for heterogeneous systems by implementing an array of schedulers for heterogeneous nodes. In addition to heterogeneous real-time systems, dynamic schedulers will be a second future research focus. We intend to expand the generality of TOPS to study various ways of incorporating sorting policies with dynamic scheduling policies. As a third future research direction, we will extend TOPS to address the issue of real-time and fault-tolerant scheduling.

\section{Acknowledgment}

Xiao Qin's work was supported by the US National Science Foundation under Grants CCF-0845257(CA-REER), CNS-0757778 (CSR), CCF-0742187 (CPA), CNS-0917137 (CSR), CNS-0831502 (CyberTrust), CNS-0855251 (CRI), OCI-0753305 (CI-TEAM), DUE-0837341 (CCLI), and DUE-0830831 (SFS). Mohammed I. Alghamdi's work was supported by Al-Baha University.

[1] Alghamdi, M. I., Jiang, X., Qin, X., Zhang, J., Jiang, M., Qiu, M., Oct 2014. Tops: Two-phase scheduling for distributed real-time systems. In: Computing, Communications and IT Applications Conference (ComComAp), 2014 IEEE. pp. 143-148. 
[2] Beaumont, O., Eyraud-Dubois, L., Caro, C. T., Rejeb, H., May 2013. Heterogeneous resource allocation under degree constraints. IEEE Transactions on Parallel and Distributed Systems 24 (5), 926-937.

[3] Bruneo, D., Distefano, S., Longo, F., Scarpa, M., Oct 2013. Stochastic evaluation of qos in service-based systems. Parallel and Distributed Systems, IEEE Transactions on 24 (10), 2090-2099.

[4] Buttazzo, G., Bertogna, M., Yao, G., Feb 2013. Limited preemptive scheduling for real-time systems. a survey. Industrial Informatics, IEEE Transactions on 9 (1), 3-15.

[5] Chen, Y.-S., Liao, H. C., Tsai, T.-H., Jan 2013. Online real-time task scheduling in heterogeneous multicore system-on-a-chip. Parallel and Distributed Systems, IEEE Transactions on 24 (1), 118-130.

[6] Chevochot, P., Puaut, I., 1999. Scheduling fault-tolerant distributed hard real-time tasks independently of the replication strategies. In: RealTime Computing Systems and Applications, 1999. RTCSA '99. Sixth International Conference on. pp. 356-363.

[7] Choudhury, P., Chakrabarti, P., Kumar, R., Jan 2012. Online scheduling of dynamic task graphs with communication and contention for multiprocessors. Parallel and Distributed Systems, IEEE Transactions on 23 (1), 126-133.

[8] Chowdhury, P., Chakrabarti, C., Feb 2005. Static task-scheduling algorithms for battery-powered dvs systems. Very Large Scale Integration (VLSI) Systems, IEEE Transactions on 13 (2), 226-237.

[9] Cichowski, P., Keller, J., May 2013. Efficient and fault-tolerant static scheduling for grids. In: Parallel and Distributed Processing Symposium Workshops PhD Forum (IPDPSW), 2013 IEEE 27th International. pp. 1439-1448.

[10] Cordasco, G., Chiara, R. D., Rosenberg, A. L., Aug 2015. An areaoriented heuristic for scheduling dags on volatile computing platforms. IEEE Transactions on Parallel and Distributed Systems 26 (8), 21642177. 
[11] Gerasoulis, A., Yang, T., Jun 1993. On the granularity and clustering of directed acyclic task graphs. Parallel and Distributed Systems, IEEE Transactions on 4 (6), 686-701.

[12] Huang, Y., Bessis, N., Norrington, P., Kuonen, P., Hirsbrunner, B., 2013. Exploring decentralized dynamic scheduling for grids and clouds using the community-aware scheduling algorithm. Future Generation Computer Systems 29 (1), 402-415.

[13] Jansen, K., Zhang, H., Jul. 2006. An approximation algorithm for scheduling malleable tasks under general precedence constraints. ACM Trans. Algorithms 2 (3), 416-434.

URL http://doi.acm.org/10.1145/1159892.1159899

[14] Kwok, Y.-K., Ahmad, I., 1999. Static scheduling algorithms for allocating directed task graphs to multiprocessors. ACM Computing Surveys (CSUR) 31 (4), 406-471.

[15] Leal, K., Huedo, E., Llorente, I. M., 2009. A decentralized model for scheduling independent tasks in federated grids. Future Generation Computer Systems 25 (8), 840-852.

[16] Lee, B., Hurson, A. R., Feng, T.-Y., 1991. A vertically layered allocation scheme for data flow systems. Journal of Parallel and Distributed Computing 11 (3), 175-187.

[17] Li, K., Tang, X., Veeravalli, B., Li, K., Jan 2015. Scheduling precedence constrained stochastic tasks on heterogeneous cluster systems. Computers, IEEE Transactions on 64 (1), 191-204.

[18] Lombardi, M., Milano, M., Benini, L., Jan 2013. Robust scheduling of task graphs under execution time uncertainty. IEEE Transactions on Computers 62 (1), 98-111.

[19] Nadiminti, K., De Assunção, M. D., Buyya, R., 2006. Distributed systems and recent innovations: Challenges and benefits. InfoNet Magazine $16(3), 1-5$.

[20] Nasri, M., Baruah, S., Fohler, G., Kargahi, M., 2014. On the optimality of $\mathrm{rm}$ and edf for non-preemptive real-time harmonic tasks. In: Proceedings of the 22nd International Conference on Real-Time Networks and Systems. ACM, p. 331. 
[21] Nasri, M., Fohler, G., 2015. Non-work-conserving scheduling of nonpreemptive hard real-time tasks based on fixed priorities. In: Proceedings of the 23rd International Conference on Real Time and Networks Systems. RTNS '15. ACM, New York, NY, USA, pp. 309-318.

URL http://doi.acm.org/10.1145/2834848.2834856

[22] Nelis, V., Andersson, B., Marinho, J., Petters, S., July 2011. Globaledf scheduling of multimode real-time systems considering mode independent tasks. In: Real-Time Systems (ECRTS), 2011 23rd Euromicro Conference on. pp. 205-214.

[23] Palmieri, F., Buonanno, L., Venticinque, S., Aversa, R., Martino, B. D., 2013. A distributed scheduling framework based on selfish autonomous agents for federated cloud environments. Future Generation Computer Systems 29 (6), $1461-1472$.

[24] Qin, X., Jiang, H., Sept 2001. Dynamic, reliability-driven scheduling of parallel real-time jobs in heterogeneous systems. In: Parallel Processing, 2001. International Conference on. pp. 113-122.

[25] Qin, X., Jiang, H., Swanson, D., 2002. An efficient fault-tolerant scheduling algorithm for real-time tasks with precedence constraints in heterogeneous systems. In: Parallel Processing, 2002. Proceedings. International Conference on. pp. 360-368.

[26] Rotithor, H., Jan 1994. Taxonomy of dynamic task scheduling schemes in distributed computing systems. Computers and Digital Techniques, IEE Proceedings - 141 (1), 1-10.

[27] Smith, J., Maciejewski, A. A., Siegel, H. J., 2014. Maximizing stochastic robustness of static resource allocations in a periodic sensor driven cluster. Future Generation Computer Systems 33, 1-10.

[28] Socci, D., Poplavko, P., Bensalem, S., Bozga, M., April 2015. Multiprocessor scheduling of precedence-constrained mixed-critical jobs. In: Real-Time Distributed Computing (ISORC), 2015 IEEE 18th International Symposium on. pp. 198-207.

[29] Stavrinides, G. L., Karatza, H. D., 2012. Scheduling real-time \{DAGs\} in heterogeneous clusters by combining imprecise computations and bin 
packing techniques for the exploitation of schedule holes. Future Generation Computer Systems 28 (7), 977 - 988.

[30] Tang, X., Li, K., Zeng, Z., Veeravalli, B., July 2011. A novel securitydriven scheduling algorithm for precedence-constrained tasks in heterogeneous distributed systems. Computers, IEEE Transactions on 60 (7), 1017-1029.

[31] Thekkilakattil, A., Dobrin, R., Punnekkat, S., July 2013. Quantifying the sub-optimality of non-preemptive real-time scheduling. In: RealTime Systems (ECRTS), 2013 25th Euromicro Conference on. pp. 113122.

[32] Venugopalan, S., Sinnen, O., Jan 2015. Ilp formulations for optimal task scheduling with communication delays on parallel systems. Parallel and Distributed Systems, IEEE Transactions on 26 (1), 142-151.

[33] Verma, A., Cherkasova, L., Campbell, R., Sept 2013. Orchestrating an ensemble of mapreduce jobs for minimizing their makespan. Dependable and Secure Computing, IEEE Transactions on 10 (5), 314-327.

[34] Xie, T., Qin, X., Sung, A., June 2005. Sarec: a security-aware scheduling strategy for real-time applications on clusters. In: Parallel Processing, 2005. ICPP 2005. International Conference on. pp. 5-12.

[35] Zhu, X., Wang, J., Wang, J., Qin, X., 2014. Analysis and design of faulttolerant scheduling for real-time tasks on earth-observation satellites. In: Parallel Processing (ICPP), 2014 43rd International Conference on. IEEE, pp. 491-500. 